C. R. Acad. Sci. Paris, t. 325, Série I, p. 511-516, 1997

Probabilités/Probability Theory

(Analyse complexe/Complex Analysis)

\title{
Non-symmetric Linnik distributions
}

\section{Mehmet Burak ERDOĞAN and Iossif Vladimirovich OSTROVSKII}

M. B. E.: Department of Mathematics, Bilkent University, 06533 Bilkent, Ankara, Turkey; E-mail: erdogan@fen.bilkent.edu.tr

I. V. O.: Department of Mathematics, Bilkent University, 06533 Bilkent, Ankara, Turkey and Verkin Institute for Low Temperature Physics and Engineering, 310164 Kharkov, Ukraine. E-mail: iossif@fen.bilkent.edu.tr, ostrovskii@ilt.kharkov.ua

Abstract. The aim of this Note is to study the probability density with characteristic function

$$
\varphi_{\alpha, \theta, \nu}(t)=1 /\left(1+e^{-\imath \theta \mathbf{g g n} t}|t|^{\alpha}\right)^{\nu}
$$

where $0<\alpha<2,|\theta| \leq \min (\pi \alpha / 2, \pi-\pi \alpha / 2)$, and $\nu>0$. This density, first introduced by Linnik for $\theta=0, \nu=1$, received several applications later. It does not have any explicit representation. We consider here its integral and series representations and its analytical properties.

\section{Les distributions de Linnik non-symmétriques}

Résumé. Nous étudions dans cette Note la densité de probabilité de fonction caractéristique

$$
\varphi_{\alpha, \theta, \nu}(t)=1 /\left(1+e^{-\imath \theta \mathbf{s g n t}}|t|^{\alpha}\right)^{\nu}
$$

où $0<\alpha<2,|\theta| \leq \min (\pi \alpha / 2, \pi-\pi \alpha / 2)$ et $\nu>0$. Cette densité, introduite pour la première fois par Linnik avec $\theta=0, \nu=1$, a trouvé quelques applications par la suite. Il n'existe aucune représentation explicite de cette densité. Nous considérons ses représentations intégrales et en séries, et ses propriétés analytiques.

\section{Version française abrégée}

En 1953, Linnik (voir [8]) a démontré que la fonction (1) est la fonction caractéristique (f.c.) correspondant à la densité de probabilité (d.p.) $p_{\alpha}$. Les propriétés de d.p. sont étudiées en détail dans [2] et [5], où on peut trouver les références concernant leurs applications. En 1984, Klebanov, Maniya et Melamed (voir [3]) ont introduit le concept de stabilité géométrique stricte, et ils ont démontré que la famille des d.p. à stabilité géométrique stricte coïncide avec la famille des d.p. dont les f.c. sont de la forme (2). Cette famille est plus vaste que la famille $\left\{p_{\alpha}: \alpha \in(0,2)\right\}$; son étude a été détaillée dans [1] (quelques résultats ont été obtenus auparavant dans [6]). Notons que pour $|\theta|=\min (\pi \alpha / 2, \pi-\pi \alpha / 2)$, les d.p. de cette famille sont apparues pour la première fois

\section{Note présentée par Jean-Pierre KaHANE.}




\section{B. Erdoğan and I. V. Ostrovskii}

dans [7] et [11]. En 1992, Pakes (voir [10]) a trouvé que les distributions de probabilité avec des f.c. de formes plus générales (3), jouent un rôle important dans des problèmes de caractérisation de statistique mathématique. L'étude des propriétés des distributions avec des f.c. (3) est donc un problème intéressant.

D'autre part, on ne peut pas appliquer les méthodes de [1], [5] et [6] à ce problème. En effet, dans le cas où $\nu=1$, la représentation (9) rend impossible la réduction du problème à l'étude d'une intégrale de type de Cauchy. Il semble qu'une telle réduction soit impossible dans le cas général où $\nu>0$. Nous utilisons donc une méthode différente, basée sur les idées de [11].

Théorème 1. - Pour les triplets (4), la distribution de probabilité avec la f.c. (3) est absolument continue, et on peut représenter sa densité $p_{\alpha, \theta, \nu}$ sous la forme (5)-(6).

Nous utilisons la représentation (5)-(6) pour étudier le développement de $p_{\alpha, \theta, \nu}$ en série et son comportement asymptotique quand $x \rightarrow 0$ et $x \rightarrow \infty$. Le théorème suivant est la base de l'étude des propriétés analytiques de $p_{\alpha, \theta, \nu}$. Notons que, par (5). et (6), il est clair que $p_{\alpha, \theta, \nu}(x)=p_{\alpha,-\theta, \nu}(-x)$. On peut donc se restreindre à l'étude des triplets (7).

TuÉréme 2. - Pour les triplets $(\alpha, \theta, \nu) \in E P D^{*}$ définis par (8), la représentation (9) est valable.

Remarque. - Les théorèmes 1 et 2 ont été démontrés dans [5] pour $\theta=0, \nu=1$, et dans [1] pour $\theta \neq 0, \nu=1$. Le théorème 2 pour $1<\alpha<2, \theta \neq 0, \nu=1$, est aussi démontré dans [4]. La représentation (10), qui n'est pas couverte par le théorème 2 , a été obtenue dans [1] pour $\theta=\pi-\pi \alpha / 2$.

Soit $[x]^{*}$ le plus grand entier strictement inférieur à $x$.

THÉOREME 3. - (i) Si $\alpha \nu \leq 2, \theta \leq \min (\pi \alpha / 2, \pi / \nu-\pi \alpha / 2)$, alors les deux fonctions $p_{\alpha, \theta, \nu}( \pm x)$ sont complètement monotones sur $(0, \infty)$. Cette assertion n'est pas valable pour toutes les valeurs de $(\alpha, \theta, \nu) \in E P D^{*}$. Dans tous les cas, pour chaque triplet $(\alpha, \theta, \nu) \in E P D^{*}$, les fonctions $p_{\alpha, \theta, \nu}( \pm x)$ sont la différence de deux fonctions complètement monotones sur $(0, \infty)$.

(ii) Si $\sin (\nu(\theta \pm \pi \alpha / 2)) \neq 0$, alors pour $k=[\alpha \nu]^{*},[\alpha \nu]^{*}+1, \ldots$, les formules (11) et (12) sont valables. Si $\theta=\pi \alpha / 2$, alors $p_{\alpha, \theta, \nu}(x)=0$ pour $x<0$.

Si $\alpha \nu>1$, alors la fonction $p_{\alpha, \theta, \nu}$ est $[\alpha \nu]^{*}-1$ fois continûment différentiable sur $\mathbb{R}$; de plus, pour $k=0,1, \ldots,[\alpha \nu]^{*}-1$, la formule (13) est valable.

Théreme 4. - Pour chaque $(\alpha, \theta, \nu) \in E P D^{*}$, les d.p. $p_{\alpha, \theta, \nu}$ sont unimodales. De plus, pour $|\theta| \leq \max (0, \pi / \nu-\pi \alpha / 2)$, le mode s'annule, et pour $|\theta|>\max (0, \pi / \nu-\pi \alpha / 2)$, le mode est positif.

Túsoreme 5. - (i) Si $(\alpha, \theta, \nu) \in E P D^{*}, \alpha \nu \leq 1$, l'égalité est valable. (ii) Comme fonction de $\theta$, $0 \leq \theta \leq \min (\pi \alpha / 2, \pi /(2 \nu)-\pi \alpha / 2), p_{\alpha, \theta, \nu}(x)$ est croissante, et $p_{\alpha, \theta, \nu}(-x)$ est décroissante pour chaque $x>0$ et $\alpha, \nu$ fixés tels que $\alpha \nu \leq 1$.

Remarque. - Le théorème 3 a été démontré pour $\theta=0, \nu=1$ dans [5], et pour $\theta \neq 0, \nu=1$ dans [1]; dans le deuxième cas, la partie (ii) a été démontrée un peu auparavant dans [6] avec quelques restrictions additionnelles. Le théorème 4 a été démontré pour $0<\alpha<2, \nu=1$ dans [1], et un peu auparavant dans [6] sous une forme plus faible. Pour le cas $\nu=1$, un résultat plus fort que le théorème 5 a été démontré dans [1].

THÉREme 6. - Pour chaque triplet $(\alpha, \theta, \nu) \in E P D$, le développement en série asymptotique (15) est valable.

Remarque. - Le thérème 6 a été démontré pour $\theta=0, \nu=1$ dans [5], pour $\theta \neq 0, \nu=1$ dans [1] et [6] et, indépendamment, sous la condition additionnelle $1<\alpha<2$ dans [4].

Les théorèmes 7,8 et 10 ci-dessous traitent du développement de $p_{\alpha, \theta, \nu}$ en séries convergentes. Pour $\nu=1$, il est démontré dans [1] et [5], que la structure de ces développements dépend de la nature arithmétique de $\alpha$. Un phénomène semblable se manifeste dans le cas général, à condition qu'on considère la nature arithmétique de deux paramètres $\alpha$ et $\nu$. 
THÉORÈm 7. - Supposons qu'une des conditions suivantes soit satisfaite : (i) $\alpha \notin \mathbb{Q}$ et $\nu \in \mathbb{Q}$; (ii) $\alpha \in \mathbb{Q}$ et $\nu \notin \mathbb{Q}$; (iii) $\alpha \notin \mathbb{Q}, \nu \notin \mathbb{Q}$ et $\nu \notin\left\{a=(2 q+1) / \alpha-p ; q \in \mathbb{Z}^{+}, p \in \mathbb{N}\right\}$; (iv) $\alpha \in \mathbb{Q}$ et $\nu \in \mathbb{Q}$, où $\alpha$ et $\nu$ sont représentables sous la forme $\alpha=m / n, \nu=k / l$ avec $m, n$ et $k, l$ des entiers relativement premiers, $l$ ne divisant pas $m$.

Alors, la représentation (16) est valable, où la limite est uniforme par rapport à $x$ sur chaque compact de $\mathbb{R}^{+}$.

Notons $L$ l'ensemble de tous les réels transcendants au sens de Liouville.

THÉRÈm 8. - Supposons qu'une des conditions suivantes soit satisfaite : (i) $\alpha \notin \mathbb{Q} \cup L$ et $\nu \in \mathbb{Q}$; (ii) $\alpha \in \mathbb{Q}$ et $\nu \notin \mathbb{Q} \cup L$; (iii) la condition (iv) du Théorème 7. Alors la représentation (17) est valable, où les termes de la partie de droite de (17) sont les mêmes que dans (16), et où les deux séries convergent absolument et uniformément par rapport à $x$ sur chaque sous-ensemble borné de $\mathrm{R}^{+}$.

Le théorème 8 n'est pas valable si on omet $L$ dans les conditions (i) ou (ii).

THÉOREME 9. - Dans l'ensemble de tous les triplets $(\alpha, \theta, \nu)$ satisfaisant les conditions (i) et (ii) du Théorème 7 , il y a un sous-ensemble dense ayant la puissance du continu pour lequel chaque série de la partie de droite de (17) est divergente pour tous les $x \in \mathbb{R}^{+}$.

Si le triplet $(\alpha, \theta, \nu)$ ne satisfait pas les conditions du théorème 7 , alors la structure du développement en séries de $p_{\alpha, \theta, \nu}$ est beaucoup plus compliquée que celle de (16) et (17). On a alors des termes en $\log x$ dans les développements.

Exemple. - Pour $\alpha=2 / 3, \theta=1, \nu=3 / 2$, la formule (18) est valable.

\section{Introduction}

In 1953, Linnik (see [8]) proved that the function

$$
\varphi_{\alpha}(t)=1 /\left(1+|t|^{\alpha}\right), \alpha \in(0,2),
$$

is the characteristic function (ch.f) of a symmetric probability density (p.d.) $p_{\alpha}$. Properties of the p.d.'s were studied in detail in [2] and [5], where one can find several references on some applications. In 1984, Klebanov, Maniya, and Melamed (see [3]) introduced the concept of geometric strict stability and proved that the family of geometrically strictly stable p.d.'s coincides with the family of p.d.'s with ch.f.'s

$$
\varphi_{\alpha, \theta}(t)=1 /\left(1+e^{-\imath \theta \text { sgnt }}|t|^{\alpha}\right), \alpha \in(0,2),|\theta| \leq \min (\pi \alpha / 2, \pi-\pi \alpha / 2) .
$$

This family is larger than $\left\{p_{\alpha}: \alpha \in(0,2)\right\}$, and its detailed study was done in [1] (some of the results had been obtained before in [6]). Note that for $|\theta|=\min (\pi \alpha / 2, \pi-\pi \alpha / 2)$, the p.d.'s of this family first appeared in [7] and [11]. In 1992, Pakes (see [10]) showed that the probability distributions with ch.f.'s more general than (2), as given in (3),

$$
\varphi_{\alpha, \theta, \nu}(t)=\frac{1}{\left(1+e^{-i \theta \text { sgnt }}|t|^{\alpha}\right)^{\nu}}, \alpha \in(0,2),|\theta| \leq \min (\pi \alpha / 2, \pi-\pi \alpha / 2), \nu>0,
$$

play an important role in characterizing some problems in mathematical statistics. Therefore, the study of properties of distributions with ch.f.'s (3) seems to be of interest.

However, the methods used in [1], [5], and [6] are not applicable to such a problem. The reason is that, in the case $\nu=1$, the representation (9) below makes it possible to reduce the problem to the study of a Cauchy type integral. Such a reduction seems to be impossible in the general case $\nu>0$. We use here different method based on ideas of [9]. 


\section{Absolute continuity and integral representations}

Theorem 1. - For any

$$
(\alpha, \theta, \nu) \in E P D:=\{(\alpha, \theta, \nu): \alpha \in(0,2),|\theta| \leq \min (\pi \alpha / 2, \pi-\pi \alpha / 2), \nu>0\},
$$

the probability distribution with ch.f.'s (3) is absolutely continuous and

$$
p_{\alpha, \theta, \nu}( \pm x)=\frac{1}{2 \pi i} \int_{c-i \infty}^{c+i \infty} f_{\alpha, \theta, \nu}^{ \pm}(z) x^{z-1} d z,-\alpha<c<\min (\alpha \nu, 1), x>0
$$

where

$$
f_{\alpha, \theta, \nu}^{ \pm}(z)=\frac{1}{\alpha \Gamma(\nu)} \frac{\sin (\pi z / 2 \pm \theta z / \alpha)}{\sin \pi z} \frac{\Gamma(z / \alpha) \Gamma(\nu-z / \alpha)}{\Gamma(z)}
$$

We use the representation (5) to study the series expansions of $p_{\alpha, \theta, \nu}$ and, hence, its asymptotic behavior as $x \rightarrow 0$ and $x \rightarrow \infty$. The next theorem is a base for studying analytical properties of $p_{\alpha, \theta, \nu}$. Note that from (5) and (6), it is obvious that $p_{\alpha, \theta, \nu}(x)=p_{\alpha,-\theta, \nu}(-x)$. Therefore, we can restrict our study to triples of the form

$$
(\alpha, \theta, \nu) \in E P D^{+}:=\{(\alpha, \theta, \nu) \in E P D: \theta \geq 0\} .
$$

THEOREM 2. - For

$$
(\alpha, \theta, \nu) \in E P D^{*}:=\left\{(\alpha, \theta, \nu) \in E P D^{+}: \theta<\pi-\pi \alpha / 2\right\}
$$

the following representation holds:

$$
p_{\alpha, \theta, \nu}( \pm x)=\frac{1}{\pi} \operatorname{Im} \int_{0}^{\infty} \frac{e^{-y x} d y}{\left(1+e^{\mp i \theta-\imath \pi \alpha / 2} y^{\alpha}\right)^{\nu}}, x>0 .
$$

Remark. - Theorems 1 and 2 had been proved in [5] for $\theta=0, \nu=1$, and in [1] for $\theta \neq 0, \nu=1$. Theorem 2 for $1<\alpha<2, \theta \neq 0, \nu=1$ is also proved in [4]. The following representation, which is not covered by Theorem 2, has been obtained in [1]:

$$
p_{\alpha, \theta, 1}(x)=-\frac{\sin (\pi \alpha)}{\pi} \int_{0}^{\infty} \frac{e^{y x} y^{\alpha} d y}{\left|1-e^{i \pi \alpha} y^{\alpha}\right|^{2}}, x<0, \quad p_{\alpha, \theta, 1}(x)=e^{-x} / \alpha, x>0
$$

for $\theta=\pi-\pi \alpha / 2$.

\section{Analytic properties of $p_{\alpha, \theta, \nu}$}

Let $[x]^{*}$ denotes the greatest integer strictly less than $x$.

THEOREM 3. - (i) If $\alpha \nu \leq 2, \theta \leq \min (\pi \alpha / 2, \pi / \nu-\pi \alpha / 2)$, then both functions $p_{\alpha, \theta, \nu}( \pm x)$ are completely monotonic on $(0, \infty)$. This statement fails to be true for all other values of $(\alpha, \theta, \nu) \in E P D^{*}$. Nevertheless, for any $(\alpha, \theta, \nu) \in E P D^{*}$, both functions $p_{\alpha, \theta, \nu}( \pm x)$ are differences of two completely monotonic functions on $(0, \infty)$.

(ii) If $\sin (\nu(\theta \pm \pi \alpha / 2)) \neq 0$, then for $k=[\alpha \nu]^{*},[\alpha \nu]^{*}+1, \ldots$, we have:

$$
\begin{gathered}
(-1)^{k+[\nu(\alpha / 2+\theta / \pi)]^{*}} \lim _{x \rightarrow 0^{+}} p_{\alpha, \theta, \nu}^{(k)}(x)=+\infty, \\
(-1)^{[\nu(\alpha / 2-\theta / \pi)]^{*}} \lim _{x \rightarrow 0^{-}} p_{\alpha, \theta, \nu}^{(k)}(x)=+\infty
\end{gathered}
$$

If $\theta=\pi \alpha / 2$, then $p_{\alpha, \theta, \nu}(x)=0$ for $x<0$. 
If $\alpha \nu>1$, then the function $p_{\alpha, \theta, \nu}$ is $[\alpha \nu]^{*}-1$ times continuously differentiable on $\mathbb{B}$ and, moreover,

$$
p_{\alpha, \theta, \nu}^{(k)}(0)=\frac{\sin [(\pi / 2-\theta / \alpha)(k+1)]}{\pi \alpha} B((k+1) / \alpha, \nu-(k+1) / \alpha)
$$

for $k=0,1, \ldots,[\alpha \nu]^{*}-1$.

THEOREM 4. - For any $(\alpha, \theta, \nu) \in E P D^{*}$, p.d.'s $p_{\alpha, \theta, \nu}$ are unimodal. Moreover, for $|\theta| \leq$ $\max (0, \pi / \nu-\pi \alpha / 2)$, the mode is zero, and for $|\theta|>\max (0, \pi / \nu-\pi \alpha / 2)$, the mode is positive.

THEOREM 5. - (i) If $(\alpha, \theta, \nu) \in E P D^{*}$ and $\alpha \nu \leq 1$, then

$$
p_{\alpha, \theta, \nu}(x) \geq p_{\alpha, \theta, \nu}(-x), x>0
$$

(ii) As a function of $\theta, 0 \leq \theta \leq \min (\pi \alpha / 2, \pi /(2 \nu)-\pi \alpha / 2), p_{\alpha, \theta, \nu}(x)$ increases and $p_{\alpha, \theta, \nu}(-x)$ decreases for any fixed $x>0$ and $\alpha, \nu$ such that $\alpha \nu \leq 1$.

Remark. - Theorem 3 has been proved for $\theta=0, \nu=1$ in [5], and for $\theta \neq 0, \nu=1$ in [1]; in the latter case, part (ii) had been proved previously in [6] under some additional restrictions. Theorem 4 has been proved for $0<\alpha<1, \nu=1$ in [6], and for $0<\alpha<2, \nu=1$ in [1]. For the case $\nu=1$, a stronger result than Theorem 5 has been proved in [1].

\section{Series expansions of $p_{\alpha, \theta, \nu}$}

THEOREM 6. - For any $(\alpha, \theta, \nu) \in E P D$,

$$
p_{\alpha, \theta, \nu}( \pm x) \sim \frac{1}{2 \Gamma(\nu)} \sum_{q=1}^{\infty} \frac{(-1)^{q} \sin (\pi \alpha q / 2 \pm \theta q) \Gamma(\nu+q)}{\sin (\pi q \alpha) \Gamma(-q \alpha) \Gamma(1+q)}|x|^{-1-q \alpha}, \quad|x| \rightarrow \infty .
$$

Remark. - Theorem 6 has been proved for $\theta=0, \nu=1$ in [5], for $\theta \neq 0, \nu=1$ in [1] and [6], and, independently, under the additional condition $1<\alpha<2$ in [4].

Theorems 7, 8, and 10 below deal with convergent series expansions of $p_{\alpha, \theta, \nu}$. For $\nu=1$ it has been shown in [1] and [5] that the structure of such expansions depends on the arithmetic nature of $\alpha$. It turns out that a similar phenomenon remains true in the general case, but one has to take into account the arithmetic nature of two parameters $\alpha$ and $\nu$.

THEOREM 7. - Suppose that one of the following conditions is satisfied:

(i) $\alpha \notin \mathbb{Q}$ and $\nu \in \mathbb{Q}$; (ii) $\alpha \in \mathbb{Q}$ and $\nu \notin \mathbb{Q}$; (iii) $\alpha \notin \mathbb{Q}, \nu \notin \mathbb{Q}$, and $\nu \notin\{a=(2 q+1) / \alpha-p$; $\left.q \in \mathbb{Z}^{+}, p \in \mathbb{N}\right\}$; (iv) $\alpha \in \mathbb{Q}$ and $\nu \in \mathbb{Q}$, where $\alpha$ and $\nu$ are representable in the form $\alpha=m / n$, $\nu=k / l$, with $m, n$ and $k, l$ relatively prime integers and $l$ not dividing $m$. Then

$$
\begin{aligned}
p_{\alpha, \theta, \nu}( \pm x)=\frac{1}{x} \lim _{s \rightarrow \infty}\{ & \frac{1}{\Gamma(\nu)} \sum_{q=0}^{s} \frac{(-1)^{q} \sin ((\nu+q)(\pi \alpha / 2 \pm \theta)) \Gamma(\nu+q)}{\sin (\pi \alpha(\nu+q)) \Gamma(\alpha(\nu+q)) \Gamma(1+q)} x^{\alpha(\nu+q)} \\
& \left.+\frac{1}{\alpha \Gamma(\nu)} \sum_{1 \leq q \leq(\nu+s+1 / 2) \alpha} \frac{(-1)^{q+1} \sin (\pi q / 2 \pm \theta q / \alpha) \Gamma(q / \alpha)}{\sin (\pi(\nu-q / \alpha)) \Gamma(q) \Gamma(1-\nu+q / \alpha)} x^{q}\right\} \\
& x>0 .
\end{aligned}
$$

This limit is uniform with respect to $x$ on every compact subset of $\mathbf{R}^{+}$.

Let us denote by $L$ the set of all Liouville transcendental numbers. 
THEOREM 8. - Suppose that one of the following conditions is satisfied: (i) $\alpha \notin \mathbb{Q} \cup L$ and $\nu \in \mathbb{Q}$; (ii) $\alpha \in Q$ and $\nu \notin Q \cup L$; (iii) is condition (iv) of Theorem 7. Then

$$
p_{\alpha, \theta, \nu}( \pm x)=\frac{1}{x \Gamma(\nu)}\left\{\sum_{q=0}^{\infty}(\ldots)+\frac{1}{\alpha} \sum_{q=1}^{\infty}(\ldots)\right\}, x>0
$$

where the summands in the right hand side of (17) are the same as in (16), with both series converging absolutely and uniformly with respect to $x$ on every compact subset of $\mathbf{R}^{+}$.

Theorem 8 fails to be true if one omits $L$ in conditions (i) or (ii).

THEOREM 9. - In the set of all $(\alpha, \theta, \nu)$ satisfying conditions (i), (ii) of Theorem 7 , there is a dense subset with the power of the continuum for which both series on the right hand side of (17) diverge for all $x \in \mathbb{R}^{+}$.

If $(\alpha, \theta, \nu)$ does not satisfy conditions of Theorem 7 , then the structure of series expansions of $p_{\alpha, \theta, \nu}$ is much more complicated than in (16) and (17). It is worth mentioning terms containing $\log x$ in the expansions.

Example. - For $\alpha=2 / 3, \theta=1, \nu=3 / 2$, we have

$$
p_{\alpha, \theta, \nu}( \pm x)=\frac{\cos (3 / 2)}{\pi} \log \frac{1}{x}+O(1), x \rightarrow+0 .
$$

The Note is the succint version of a text on file for five years in the Academy Archives. Copy available upon request.

Note remise le 18 mars 1997 , acceptée le 18 juin 1997.

\section{References}

[1] Erdogan B., 1995. Analytic and asymptotic properties of non-symmetric Linnik's probability densities, Thesis, Bilkent University, Ankara.

[2] Ilayfavi A., Kotz S., Ostrovskii I. V., 1994. Analytic and Asymptotic Properties of Linnik's Probability Densities, $C$. $R$. Acad. Sci. Paris, 319, Série I, pp. 985-990.

[3] Klubanov L. B., Maniya G. M. and Melamed J. A., 1984. A problem of Zolotarev and analogs of infinitely divisible and stable distributions in a scheme for summing a random number of random variables, Theor. Probab. Appl., $29, \mathrm{pp} .791-794$.

[4] Klebanov L. B., Melamed J. A., Mittnik S. and Rachev S. T., 1996. Integral and asymptotic representations of geo-stable densities, Appl. Math. Lett., 9, no. 6, pp. 37-40.

[5] Kotz S., Ostrovskii I. V. and Ilayfavi A., 1995. Analytic and Asymptotic Properties of Linnik's Probability Densities, I. II, J. Math. Anal. Appl., 193, pp. 353-371, 497-521.

[6] Kozubowskl T.J., 1994. Representation and properties of geometric stable laws. In Approximation Probability, Related Fields (eds. G. Anastassiou and S. T. Rachev) Plenum Press, New York, 1994, pp. 321-337.

[7] Laha R. G., 1961. On a class of unimodal distributions, Proc. Amer. Math. Soc., 12, pp. 181-184.

[8] Linnik Yu. V., 1963. Linear forms and statistical criteria, I, II, Selected Translations in Mathematical Statistics and Probability, 3. pp. 1-90. (Original paper appeared in: Ukrainskii Mat. Zhournal, 5, 1953, pp. 207-209.)

[9] Ostrovskii I. V., 1995. Analytic and asymptotic properties of multivariate Linnik's distribution, Math. Phys., Anal. Geom., 2. pp. 436-455.

[10] Pakes A. G., 1992. A characterization of gamma mixtures of stable laws motivated by limit theorems, Stat. Neerlandica, 2-3. pp. 209-218.

[11] Pillal R. N., 1990. On Mittag-Leffler functions and related distributions, Ann. I. Stat. Math., 42, pp. 157-161. 